\title{
Single-Molecule Translocation Conformational Sensing of Multi-Arm DNA Concatemers Using Glass Capillary Nanopore
}

\author{
Ya Zhou ${ }^{\dagger, \ddagger}$, Ruiping $\mathrm{Wu}^{+, \ddagger}$, Dandan Wang ${ }^{\dagger, \ddagger}$, Ping $\mathrm{Hu}^{\dagger, \ddagger}$, Yongdong Jin* $*^{\dagger, \neq}$ \\ 'State Key Lab. of Electroanalytical Chemistry, Changchun Institute of Applied \\ Chemistry, Chinese Academy of Science, Changchun, 130022, China. \\ ${ }^{\ddagger}$ University of Science and Technology of China, Hefei, 230026, China
}

Email: ydjin@ciac.ac.cn 


\section{Contents}

Experimental Section...........................................................

The synthesis of the multiple-arm concatemers........................4

The preparation and size calculation of glass nanopores............6

The translocation process for the $10 \mathrm{kbp}$ DNA maker...............7

The translocation process for one-arm DNA concatemers.........7

The translocation process for two-arm DNA concatemers..........8

The characteristic signal for three-arm and four-arm DNA

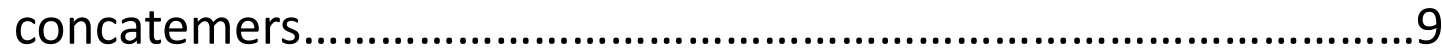

The translocation events for three-arm DNA concatemers in glass

nanopore with different diameters...........................................

The calculation of the translocation frequency for multi-arm DNA

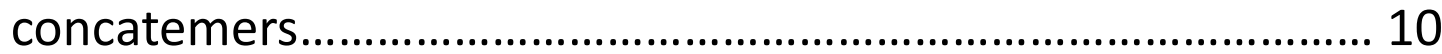

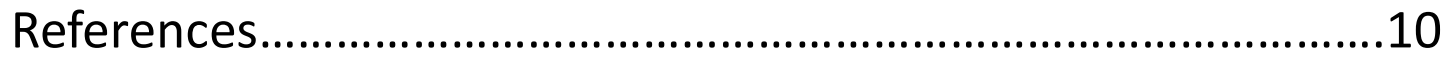




\section{Experimental section:}

The preparation of glass nanopores. Glass capillary nanopores were fabricated with quartz glass capillary (Sutter Instrument Co.). Its external diameter is 1 $\mathrm{mm}$ and inner diameter is $0.7 \mathrm{~mm}$. All glass capillaries were immersed in freshly prepared piranha solution $\left(3: 198 \% \mathrm{H}_{2} \mathrm{SO}_{4} / 30 \% \mathrm{H}_{2} \mathrm{O}_{2}\right)$ for $\sim 2 \mathrm{~h}$ to remove organic impurities. (Caution: piranha solution is a dangerous agent which reacts violently with organic compounds. It should be handled with extreme care.) The capillaries were washed thoroughly with deionized water and vacuum dried at $80{ }^{\circ} \mathrm{C}$ before use. The glass nanopores were then fabricated with $\mathrm{CO}_{2}$-laser-actuated pipette puller (model P2000, Sutter Instrument Co.) with an one-line program including the following parameters (Table S2).

The synthesis of the multi-arm DNA concatemers. $\mathrm{H} 1$, and $\mathrm{H} 2$ were

diluted in $1 \times$ TNaK (20 mM Tris- $\mathrm{HCl}, 140 \mathrm{mM} \mathrm{NaCl}, 5 \mathrm{mM} \mathrm{KCl}, \mathrm{pH}$ 7.5) for reactions. Linker and $A 1, A 2, A 3$ were pre-annealed to form four types of multiple-arm linked initiators. $\mathrm{H} 1$ and $\mathrm{H} 2$ were then respectively annealed at $95{ }^{\circ} \mathrm{C}$ for $12 \mathrm{~min}$ and cooled down to $25{ }^{\circ} \mathrm{C}$ at a rate of $0.1{ }^{\circ} \mathrm{C} / \mathrm{s}$ before use. To start reaction, $10 \mu \mathrm{L}$ of $\mathrm{H} 1,10 \mu \mathrm{L}$ of $\mathrm{H} 2,10 \mu \mathrm{L}$ of $2 \mathrm{M} \mathrm{NaCl}$, and $10 \mu \mathrm{L}$ of different multiple-arm linked initiators were mixed together, forming a standard $40 \mu \mathrm{L}$ reaction liquid. After the liquid was incubated at $16{ }^{\circ} \mathrm{C}$ for at least $12 \mathrm{~h}, 33 \mu \mathrm{L}$ of reaction product was used for electrochemistry measurement and $5 \mu \mathrm{L}$ of it was loaded into agarose gel for electrophoresis test. The $2 \%$ agarose gels contained $0.1 \mu \mathrm{L}$ Gel red per $\mathrm{ml}$ of gel volume and were prepared by using $1 \times$ TAE buffer. Agarose gels were run at $120 \mathrm{~V}$ for $30 \mathrm{~min}$ and visualized under UV light.

DNA translocation studies. For DNA translocation, the concatemers were put out of the glass nanopore. The circuit system is connected by two silver-silver chloride electrodes placed in an electrolyte solution, one of which acts as a reference electrode in an external solution, and the other silver-silver chloride electrode in the nanopore as a working electrode applying an electric field to the sensing zone of the nanopipette. The current signals were collected with current amplifier Axopatch 200B (Molecular Devices) using a lowpass Bassel filter of $10 \mathrm{kHz}$ and digitized with a DigiData 1440A digitizer (Molecular Devices) at a sample rate of $100 \mathrm{kHz}$. The translocations of DNA concatemer were recorded in $25 \mathrm{mM}$ Tris- $\mathrm{HCl}, 640 \mathrm{mM} \mathrm{NaCl}, 5 \mathrm{mM} \mathrm{KCl}$, and $0.5 \mathrm{mM}$ EDTA, pH 7.5. The current signal is analyzed with Clampfit 10.7 software (Molecular Devices). 


\section{The synthesis of the multiple-arm concatemers.}

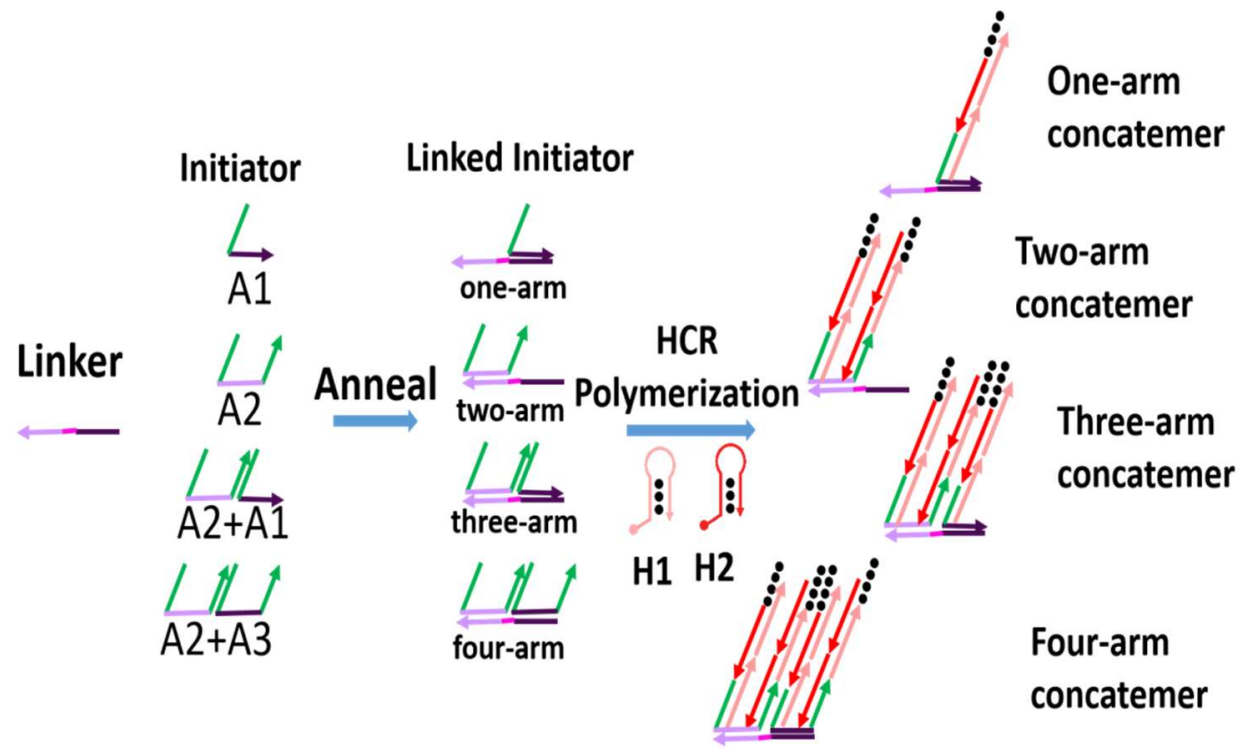

Figure S1. The schematic diagram of the synthesis steps of the multiple-arm concatemer.

\begin{tabular}{|c|l|}
\hline Linker & $\begin{array}{l}\text { GCCGAGGTGAAGTTCGAGGGCGACACCCTGGTGAAC } \\
\text { CGCATCGAGCTGAAAAAAAAGGCATCAAGGTGAACT } \\
\text { TCAAGATCCGCCACAACATCGAGGACGGCAGCGT }\end{array}$ \\
\hline $\mathbf{A 1}$ & CCTCGTAAATCCTCATCAATCATCCAGTAAACCGCC \\
& AAAAAACGCTGCCGTCCTCGATGTTGTGGCGGATCTT \\
& GAAGTTCACCTTGATGCC \\
\hline $\mathbf{A 2}$ & CCTCGTAAATCCTCATCAATCATCCAGTAAACCGCC \\
& AAAAATCAGCTCGATGCGGTTCACCAGGGTGTCGCC \\
& CTCGAACTTCACCTCGGCAAAAAAGCTCAGTCCATCC \\
& TCGTAAATCCTCATCAATCATC \\
\hline $\mathbf{A 3}$ & CCTCGTAAATCCTCATCAATCATCCAGTAAACCGCC \\
& AAAAAACGCTGCCGTCCTCGATGTTGTGGCGGATCTT \\
& GAAGTTCACCTTGATGCCAAAAAAGCTCAGTCCATCC \\
& TCGTAAATCCTCATCAATCATC \\
\hline $\mathbf{H 1}$ & GGCGGTTTACTGGATGATTGATGAGGATTTACGAGG \\
& AGCTCAGTCCATCCTCGTAAATCCTCATCAATCATC \\
\hline $\mathbf{H 2}$ & CCTCGTAAATCCTCATCAATCATCCAGTAAACCGCCG \\
& ATGATTGATGAGGATTTACGAGGATGGACTGAGCT \\
\hline
\end{tabular}

Table S1. Oligonucleotides used in this paper. 


\section{The preparation and size calculation of glass nanopores.}

We can use the electrochemical measurement to estimate the nanopore diameter according to the classical equation.s1

$$
\mathrm{a}=\frac{1}{\pi \kappa \tan \theta / 2}
$$

Where $R$ is the measured pipette resistance, $\kappa$ is the specific resistance of the electrolyte used ( $\kappa=1.2 \mathrm{~S} / \mathrm{m}$ in $0.1 \mathrm{M} \mathrm{KCl}), \theta$ is the cone angle and $a$ is the radius of the nanopore at the tip of the nanopipette.

The calculated pore size was in good agreement with that obtained with TEM image $(15 \pm 5 \mathrm{~nm})$ based on the measurements using six randomly selected nanopipettes.

To minimize the calculation error, we chose the current data between - 50 $\mathrm{mV}$ and $50 \mathrm{mV}$ because in this interval the linear and rectification ratio is close to 0 . We measured six nanopipettes and calculated their average value. In our system, the pore size is about $15 \pm 5 \mathrm{~nm}$, and the error is $30 \%$ due to the small size of the nanopore. Although the sensitive current-voltage rectification showed a large variation due to uncontrollable sensing interface of the nanopore, it does not affect the translocation studies of the nanopore.

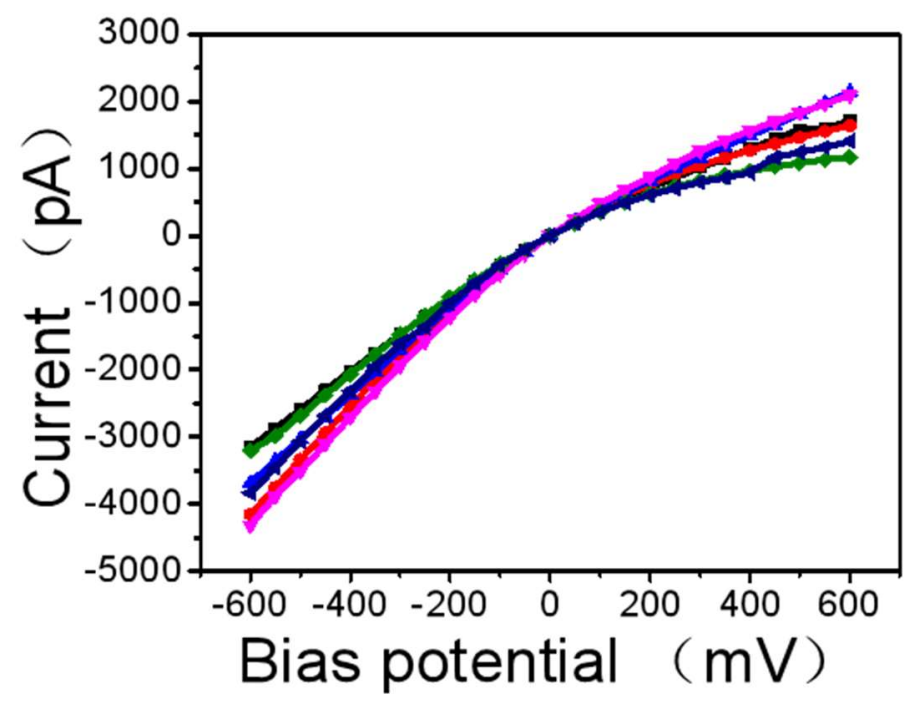

Figure S2. The I-V curves of the glass nanopores (with orifice size of $\sim 15 \mathrm{~nm}$ ) measured for six nanopipettes with the same program 1 at the buffer solution. 
Table S2. Programs (different parameters) of P-2000 laser-based micropipette puller used for fabricating glass nanopores with different diameters used in this study.

\begin{tabular}{|c|c|c|c|c|c|c|c|}
\hline Program & Cycle & Heat & Fil & Vel & Del & Pull & $\mathrm{d}(\mathrm{nm})$ \\
\hline 1 & 1 & 760 & 4 & 29 & 140 & 168 & 15 \\
\hline 2 & 1 & 700 & 4 & 60 & 170 & 180 & 30 \\
\hline 3 & 1 & 700 & 3 & 40 & 175 & 190 & 50 \\
\hline \multirow{2}{*}{$4^{*}$} & 1 & 350 & 3 & 30 & 220 & 0 & 100 \\
\cline { 2 - 7 } & 2 & 340 & 2 & 27 & 180 & 250 & \\
\hline
\end{tabular}

* Borosilicate capillaries were used to fabricate nanopore with large size in Program 4, while quartz capillaries were used in other programs.

\section{The translocation process for the $10 \mathrm{kbp}$ DNA maker.}

a

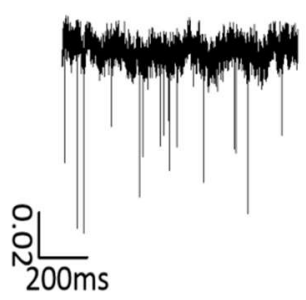

b

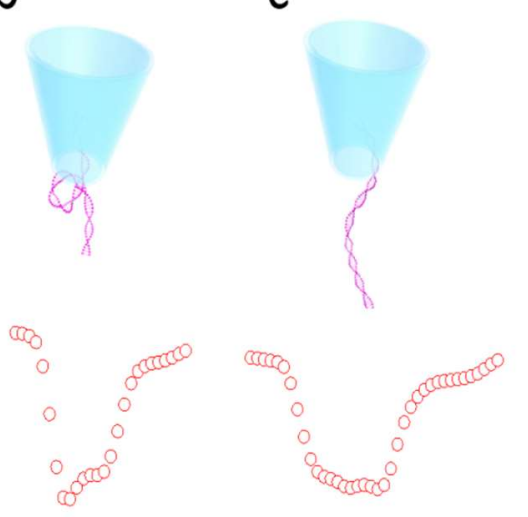

d

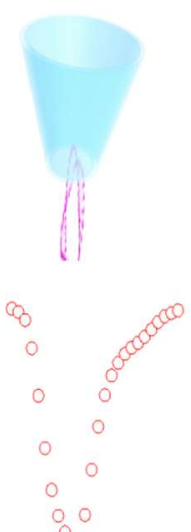

인ㄴ

Figure S3. The schematic diagram of the translocation process for the $10 \mathrm{kbp}$ DNA maker. (a) The raw current trace recoded in $1 \mathrm{~s}$. (b-d) Typical current-time traces for $10 \mathrm{kbp}$ maker translocating through the glass nanopore (with orifice size of $15 \mathrm{~nm}$ ) at $1 \mathrm{~V}$ at different conformational states: partial folded (b), linear (c), folded (d) in buffer solution. 

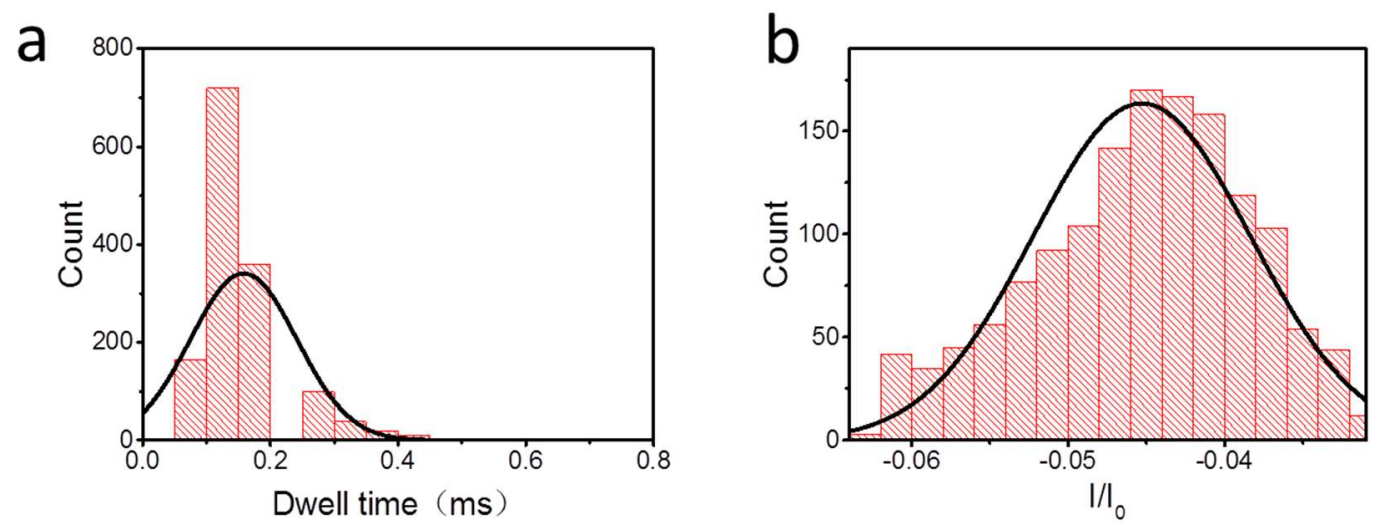

Figure S4. The dwell time (a) and current drop (b) histogram distribution for the 10 kbp DNA marker translocating through a glass nanopore (with orifice size of $\sim 15 \mathrm{~nm}$ ) at $1 \mathrm{~V}$ in the buffer solution.

\section{The translocation process for one-arm DNA concatemers.}

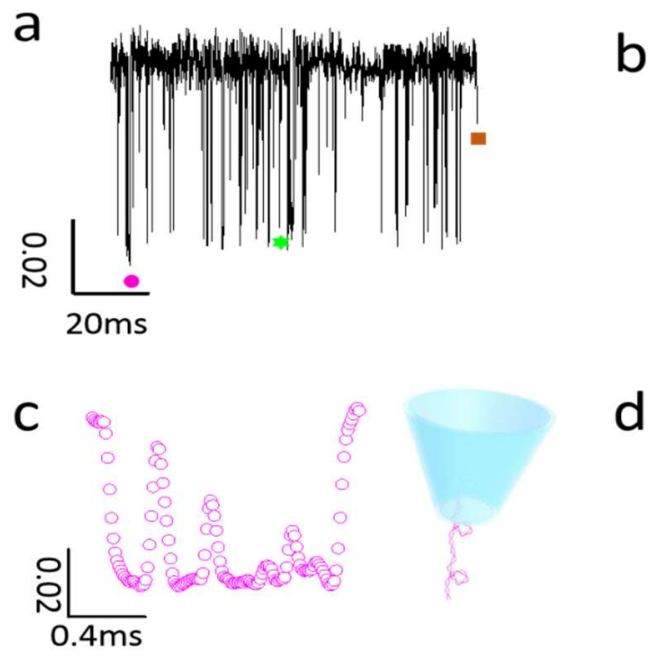

b

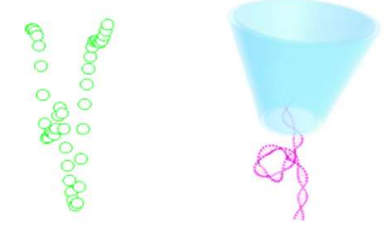

d

Figure S5. (a) The raw current trace recorded in $100 \mathrm{~ms}$. (b-d) The schematic diagram of the one-arm DNA concatemers passing through the glass nanopore (with orifice size of $\sim 15 \mathrm{~nm}$ ) at $1 \mathrm{~V}$ in the buffer solution at different conformational states: partially folded (b), knotted (c), linear double strands (d). 


\section{The translocation process for two-arm DNA concatemers.}

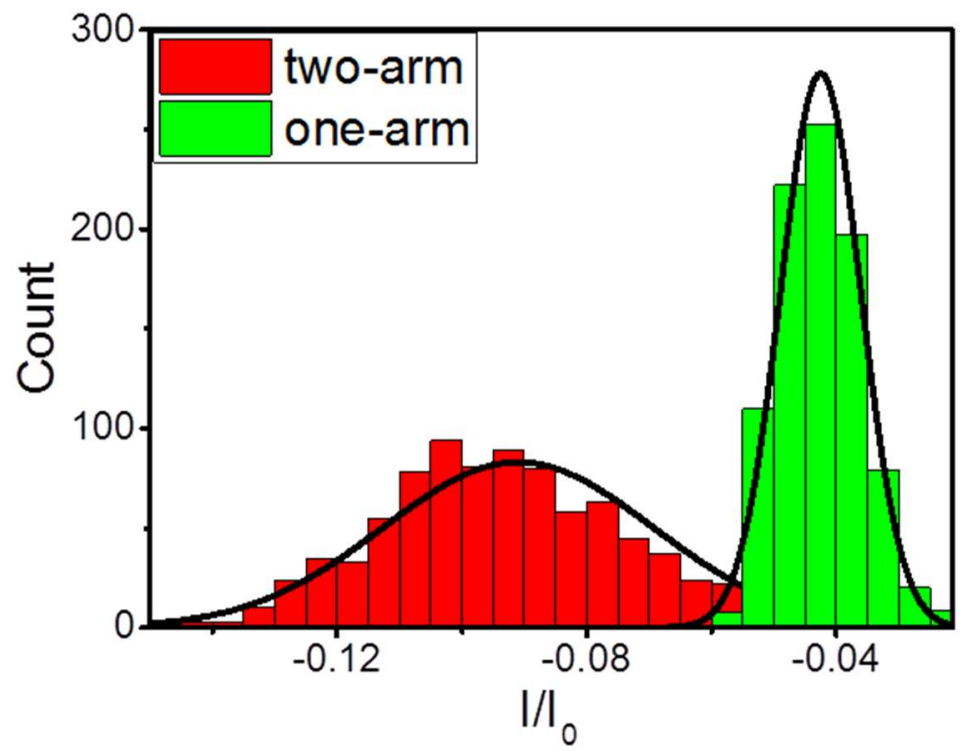

Figure S6. The typical current drop histogram distribution for one-arm and two-arm DNA concatemers translocating through a glass nanopore (with orifice size of $\sim 15$ $\mathrm{nm})$ at $1 \mathrm{~V}$ in the buffer solution.
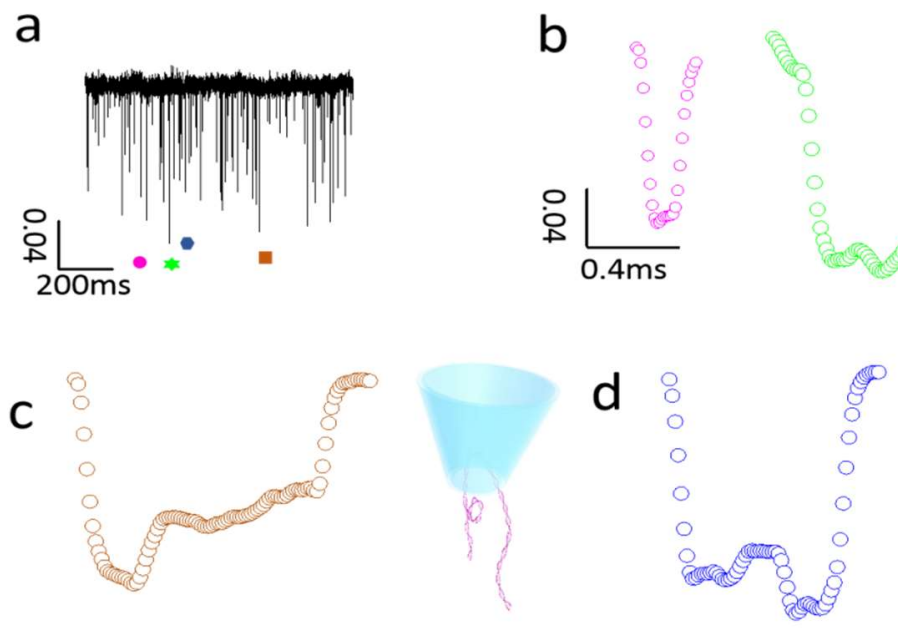

Figure S7. (a) The raw current trace recorded in $1 \mathrm{~s}$. (b-d) The schematic diagram of the two-arm DNA concatemers passing through the glass nanopore (with orifice size of $\sim 15 \mathrm{~nm}$ ) at $1 \mathrm{~V}$ in the buffer solution at different conformational states: knotted (b), single arm knotted (c), two arm knotted (d). 


\section{The characteristic signal for partially folded three-arm and four-arm DNA concatemers.}
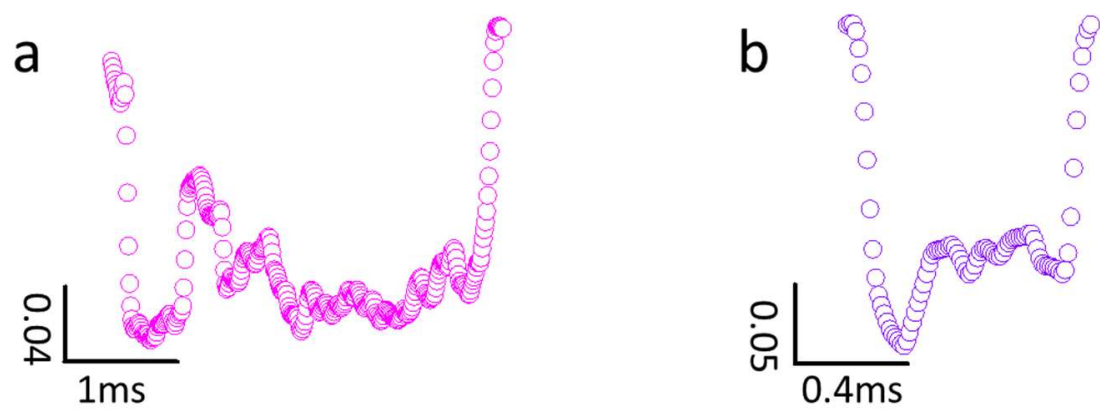

Figure S8. The characteristic current profile of the three-arm (a) and four-arm (b) DNA concatemers at partially folded state.

\section{The translocation events of three-arm DNA concatemers through glass nanopore with different diameters.}

a

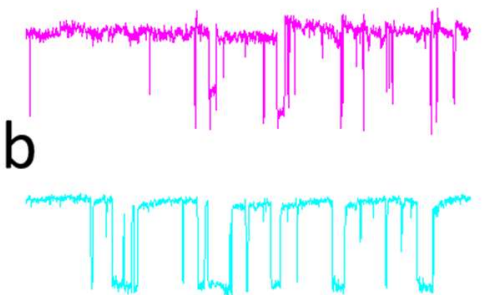

C

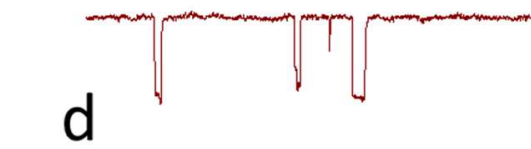

e

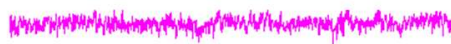<smiles>[3H][3H]</smiles>

g

$\mathrm{h}$
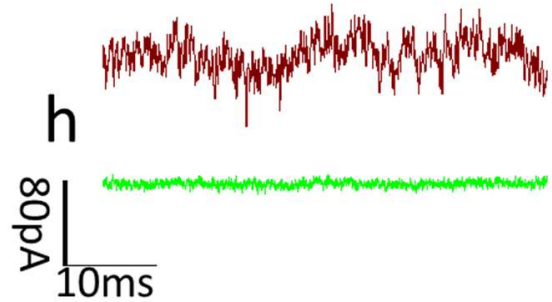

Figure S9. The raw current traces (left) and corresponding baselines (right) for the three-arm DNA concatemers translocating through glass nanopores with four different diameters: $(a, e) 15 \mathrm{~nm}$ nanopore, at the potential of $1 \mathrm{~V}$; $(\mathrm{b}, \mathrm{f}) 30 \mathrm{~nm}$ nanopore, at the potential of $1 \mathrm{~V}$; $(c, g) 50 \mathrm{~nm}$ nanopore, at the potential of $1 \mathrm{~V} ;(\mathrm{d}, \mathrm{h}) 100 \mathrm{~nm}$ nanopore, at the potential of $200 \mathrm{mV}$ (when the bias potential set as $1 \mathrm{~V}$, the current is out of range). The $y$ axis represents the current drop $\left(1 / I_{0}\right)$, and the $x$ axis is the time scale. 


\section{Theoretical estimation of the translocation frequency for multi-arm DNA concatemers.}

DNA translocation frequency depends on three steps: ( I ) DNA diffusion to the mouth of nanopore; ( II ) DNA capture by the nanopore; and (III) DNA pass through the nanopore. ${ }^{\mathrm{s} 2}$

$$
\mathrm{J}(\mathrm{x}, \mathrm{t})=-\mathrm{D} \frac{\partial c(x, t)}{\partial x}-\mathrm{c}(\mathrm{x}, \mathrm{t}) \mu \frac{\partial \mathrm{V}(\mathrm{x})}{\partial x}-\mathrm{Dc}(\mathrm{x}, \mathrm{t}) / \mathrm{kT} \frac{\partial \mathrm{F}(\mathrm{x})}{\partial x},
$$

where $D$ is the diffusion coefficient, $x$ is the center of mass coordinate of the DNA, $c(x, t)$ is the concentration, $\mu$ is the electrophoretic mobility, $V(x)$ is the electric potential, $T$ is the temperature, and $F(x)$ is the entropic barrier. When electrophoresis forces play a dominant role in DNA translocation, such as single double-stranded DNA translocation, the equation can be simplified to

$$
J(x, t)=c(x, t) \mu \frac{\partial v(x)}{\partial x}
$$

If the concentration of DNA is $C_{0}$ and the voltage is uniformly distributed in the sensing region, then

$$
\mathrm{J}(\mathrm{L})=\mathrm{C}_{0} \mu \mathrm{V}_{\mathrm{m}} / \mathrm{L}, \quad \mu=\mathrm{zD} / \mathrm{kT}
$$

In this case, the translocation frequency of the DNA concatemers is only related to the applied electric field. However, due to the complex structure of the DNA concatemers, the entropic barrier is much higher than linear DNA molecules. Therefore, the translocation frequency of DNA concatemers is caused by the competition between electrophoretic force and entropic barrier.

\section{References:}

S1. Fu, Y.; Tokuhisa, H.; Baker, L. A. Nanopore DNA sensors based on dendrimer-modified nanopipettes. Chem.Commun. 2009, 4877-4879.

S2. Bell, N. A. W.; Muthukumar, M.; Keyser, U. F. Translocation frequency of double-stranded DNA through a solid-state nanopore. Phys. Rev. E. 2016, 022401. 